\title{
Tripterine Treatment Improves Endothelial Progenitor Cell Function via Integrin- Linked Kinase
}

\author{
Chenhui Lu ${ }^{\mathrm{a}}$ Xixiang Yu ${ }^{\mathrm{b}}$ Keqiang Zuo ${ }^{\mathrm{a}}$ Xiaoping Zhang ${ }^{\mathrm{a}, \mathrm{c}}$ Chuanwu Cao ${ }^{\mathrm{a}}$ \\ Jichong Xua Shi Wang ${ }^{a}$ Tao Tang ${ }^{a}$ Meng Ye $^{a}$ Erli Pei ${ }^{a}$ Georges Uzan ${ }^{d}$ \\ Kangkang Zhi ${ }^{\mathrm{e}}$ Maoquan Li ${ }^{\mathrm{a}, \mathrm{c}}$ \\ aDepartment of Interventional and Vascular Surgery, Shanghai Tenth People's Hospital, Tongji \\ University, Shanghai, China; ${ }^{b}$ Department of Interventional Radiology, the Third Affiliated Hospital of \\ Wenzhou Medical University, Ruian, China; 'Institute of Intervention Vessel, Tongji University, Shanghai, \\ China; dUnite de Recherche INSERM 972, Paris, France; eDepartment of Vascular and Endovascular \\ Surgery, Changzheng Hospital, Shanghai, China
}

\section{Key Words}

Atherosclerosis • Endothelial progenitor cells • Tripterine $\bullet$ Integrin-linked kinase

\begin{abstract}
Background/Aims: Atherosclerosis is associated with dysfunction of endothelial progenitor cells (EPCS). Tripterine, a chemical compound derived from the Chinese medicinal plant Tripterygium wilfordii Hook, displays anti-inflammatory properties in several animal models. We hypothesized that tripterine can improve EPC function and thus the efficiency of EPC transplantation. Methods and Results: Tripterine preconditioning $(2.5 \mu \mathrm{M}, 4 \mathrm{~h})$ improved EPC proliferation, tube formation, migration, and adhesion, and reduced apoptosis in cells cultured in ox-LDL $(200 \mu \mathrm{g} / \mathrm{ml})$. Tripterine restored integrin-linked kinase (ILK) levels downregulated by ox-LDL in EPCs, suggesting the involvement of the ILK/Akt pathway. Small interfering RNA-mediated depletion of ILK and dominant-negative ILK transduction inhibited the phosphorylation of the ILK downstream signaling targets protein kinase B/ Akt and glycogen synthase kinase 3-beta (GSK-3ß), and reduced $\beta$-catenin and cyclin D1 expression. In atherosclerotic mice injected with green fluorescent protein-labeled EPCs to evaluate EPC function, tripterine decreased aortic lesions and plaque deposition, and injection of tripterine-treated EPCs restored ILK levels. Conclusion: The present results suggest that tripterine improves vascular function in atherosclerosis by enhancing EPC function through a mechanism involving the ILK signaling pathway.
\end{abstract}

C. Lu, X. Yu and K. Zuo contributed equally to this work.

Kangkang Zhi and Maoquan Li

KARGER 125
Department of Vascular and Endovascular Surgery, Changzheng Hospital, 415 Fengyang Road, Shanghai, (China); and Department of Interventional and Vascular Surgery, Shanghai Tenth People's Hospital, Tongji University, No. 301, Yanchang Road, Shanghai, (China) Institute of Intervention Vessel, Tongji University, North Zhongshan Road, Shanghai, (China); E-Mail_kangkang_zhi@163.com, E-Mail cjr.limaoquan@vip.163.com 


\section{Introduction}

Atherosclerosis results from the deposition of low-density lipoprotein (LDL) in the vascular subendothelium and the consequent influx of inflammatory leukocytes into the vascular wall leading to the development of atherosclerotic plaque. It is associated with several cardiovascular diseases and is a major cause of mortality worldwide [1]. Endothelial progenitor cells (EPCs) derived from the bone marrow play an important role in tissue regeneration, in particular in vascular repair, by homing to sites of endothelial damage, where they differentiate into mature endothelial cells and become incorporated into the endothelial lining, promoting neovascularization and endothelial regeneration $[2,3]$. EPCs produce angiogenic cytokines and growth factors that promote the proliferation of local endothelial cells and activate angiogenesis, resulting in the re-establishment of endothelial homeostasis $[4,5]$. The transplantation of mononuclear bone marrow derived or cultured EPCs has been developed as a therapeutic strategy for the treatment of ischemic diseases, and intramyocardial transplantation of autologous EPCs attenuates myocardial ischemia [68]. However, the efficiency of EPC transplantation is limited by the functional integrity of EPCs, and the development of strategies to improve EPC function is an active area of research.

Integrin-linked kinase (ILK) is a conserved 59-kDa multidomain serine-threonine kinase that links the actin cytoskeleton to the extracellular matrix by binding directly to integrins, thus playing an important role in cell migration [9]. ILK interacts with $\beta 1$ and $\beta 3$ integrins, lipid second messengers, Akt and GSK-3, and adaptor proteins such as PINCH-1 and -2 , paxillin, and $\alpha$ - and $\beta$-parvin [10]. The interaction of ILK with $\beta 1$ and $\beta 3$ integrins increases the kinase activity of ILK, resulting in the phosphorylation of GSK-3 and Akt, a mechanism that has been implicated in integrin-mediated survival and regulation of gene expression [11]. ILK regulates several cellular functions including proliferation, survival, differentiation, adhesion and migration [12-14]. The ILK-dependent phosphorylation of GSK-3 $\beta$ inhibits the activity of the pro-apoptotic protein Bim.

Tripterine, also known as celastrol, is derived from the traditional Chinese medicinal plant Trypterygium wilfordii. It is a pentacyclic triterpene with known anti-inflammatory activity mediated by the inhibition of the expression of inflammatory genes including interleukin-1 $\beta$, tumor necrosis factor- $\alpha$, prostaglandin E2 and inducible nitric oxide (NO) synthase [15]. Tripterine inhibits the synthesis of peroxynitrite precursors, which are produced by endothelial cells in response to pro-inflammatory stimuli, and is thus implicated in the protection against endothelial barrier dysfunction. Tripterine was shown to inhibit atherosclerotic plaque development in a mouse model through the inhibition of the lectinlike ox-LDL receptor LOX-1 and the reduction of oxidative stress [16]. The atheroprotective effects of tripterine were demonstrated in a recent study investigating the inhibition of resistin induced smooth muscle cell (SMC) migration, which is associated with vascular restenosis [17].

Inapreviousstudy from ourgroup, we showed thattripterineimprovesneovascularization by promoting EPC mobilization and migration and inducing vasculogenesis through a mechanism involving the heat shock protein HSP32 and inhibition of pro-apoptotic signaling pathways [18]. In the present study, we further investigated the effects of tripterine on the function of EPCs in vitro and in vivo. Our results showed that tripterine improves neovascularization, proliferation, migration, and adhesion and suppresses apoptosis in EPCs via a mechanism involving ILK and its downstream effectors Akt and GSK-3 $\beta$.

\section{Materials and Methods}

Mouse EPC isolation and cultivation

EPCs were isolated from mouse bone marrow. Briefly, mononuclear cells were separated from the tibia and femur of male 8 week old C57BL/6J mice by Ficoll density gradient centrifugation and cultured on human fibronectin-coated plates in endothelial cell growth media (EGM-2) supplemented with EGM ${ }^{\mathrm{TM}}-2 \mathrm{MV}^{\mathrm{M}}$ 
SingleQuots ${ }^{\mathrm{TM}}$ (Lonza, Basel, Switzerland), mouse recombinant vascular epidermal growth factor (VEGF; 20 $\mathrm{ng} / \mathrm{ml}$ ), insulin-like growth factor ( $4 \mathrm{ng} / \mathrm{ml}$ ), fibroblast growth factor $(4 \mathrm{ng} / \mathrm{ml}$ ) and $5 \%$ fetal bovine serum. After 4 days of culture in a $5 \% \mathrm{CO}_{2}$ incubator, non-adherent cells were removed by washing with phosphatebuffered saline (PBS) and adherent cells were incubated in fresh media for a further 3 days for the following experiments.

\section{EPC characterization}

The identity of EPCs was confirmed using a phase-contrast microscope (IX71; Olympus; Tokyo, Japan). EPCs were characterized as adherent cells double positive for acetylated low-density lipoprotein uptake and Ulex europaeus lectin binding by fluorescent microscopy. The cells were incubated with $2.4 \mu \mathrm{g} / \mathrm{ml} 1$, 1'-dioctadecyl-3,3,3',3'-tetramethylindocarbocyanine perchlorate-acetylated low-density lipoprotein (DiIacLDL; Molecular Probes, Eugene, OR) for $1 \mathrm{~h}$, fixed in 2\% paraformaldehyde and counterstained with 10 $\mu \mathrm{g} / \mathrm{ml}$ FITC-labeled lectin from Ulex europaeus (UEA-1) (Sigma). The fluorescent images were recorded under a laser scanning confocal microscope.

\section{Preparation of ox-LDL and cell treatment}

To generate oxidized LDL (ox-LDL), native LDL was incubated with $\mathrm{CuSO}_{4}(10 \mu \mathrm{M})$ at $37^{\circ} \mathrm{C}$ for $24 \mathrm{~h}$, dialyzed against a sterile solution of $\mathrm{NaCl}(150 \mathrm{mM})$ EDTA $(1 \mathrm{mM})$ and polymixin B $(100 \mu \mathrm{g} / \mathrm{ml})(\mathrm{pH}$ 7.4). The presence of ox-LDL was confirmed by agarose gel electrophoresis and generation of thiobarbituric acid. To determine the optimal concentration and time of ox-LDL treatment, preliminary experiments were performed by incubating EPCs with different concentrations of ox-LDL for different times and its effect on the formation of adherent cell colonies was determined.

\section{EPC proliferation and adhesion}

EPC proliferation was assessed by measuring DNA synthesis using the 5-ethynyl-2-deoxyuridine (EdU) incorporation assay and the Click-iT EdU Alexa Fluor 488 Imaging kit (Invitrogen). Cells were labeled with EdU $(10 \mu \mathrm{M})$ for $6 \mathrm{~h}$ and fixed with 3.7\% formaldehyde in PBS for $15 \mathrm{~min}$ at room temperature followed by 0.5\% Triton X-100 in PBS for 20 min at room temperature. After incubation with Click-iT reaction cocktail and Hoechst 33342 solution for $30 \mathrm{~min}$ each, cells were imaged using a fluorescence microscope. EPC adhesion assay was performed by culturing cells on fibronectin-coated 6-well plates for 30 min at $37^{\circ} \mathrm{C}$. After washing with PBS 3 times, adherent cells were counted and phenotyped as described above.

As an additional approach, cell proliferation was assessed by immunocytochemical staining for Ki67 using an anti-Ki67 primary antibody (Abcam) and a FITC-coupled secondary antibody (Invitrogen). Nuclei were stained with DAPI.

\section{Tube formation assay}

EPC tube formation assay was performed using the In Vitro Angiogenesis Assay kit (Chemicon). The ECMatrix gel solution was thawed overnight at $4^{\circ} \mathrm{C}$, mixed with ECMatrix diluent buffer and placed in a 96well plate for $1 \mathrm{~h}$ at $37^{\circ} \mathrm{C}$. EPCs were harvested and $1 \times 10^{4} \mathrm{EPCs}$ were placed onto a matrix solution with EGM-2 MV medium and incubated at $37^{\circ} \mathrm{C}$ for $16 \mathrm{~h}$. Tube formation was visualized using an inverted light microscope and the number of tubules formed per high power field was determined using Image-Pro Plus software.

\section{Apoptosis assays}

The terminal deoxynucleotidyl transferase-mediated deoxyuridine triphosphate nick-end labeling (TUNEL) assay was performed using the In Situ Cell Death Detection kit (Roche Diagnostics, Mannheim, Germany) according to the manufacturer's instructions. EPCs were counterstained with 4', 6-diaminophenylindole (DAPI). Apoptosis was determined as the percentage of TUNEL positive cells per 1000 DAPI stained nuclei after visualization under a Olympus BX50 fluorescence microscope at a magnification of 100x.

As an additional approach, cleaved (active) caspase-3 immunocytochemistry was performed using standard protocols. Nuclei were stained with DAPI. The expression of cleaved caspase-3 was determined using a specific antibody, followed by a secondary antibody conjugated with FITC.

\section{KARGER}




\section{Cell migration assay}

EPC migratory ability was assessed using a modified Boyden chamber assay. Isolated EPCs were detached with trypsin/EDTA and $4 \times 10^{4}$ cells were placed in the upper chambers of 24-well Transwell plates with $8 \mu \mathrm{m}$ pore polycarbonate membrane inserts in serum free growth medium. The medium in the lower chambers was supplemented with VEGF (50 ng/ml). After $24 \mathrm{~h}$ of incubation, the membrane was washed with PBS and fixed with 4\% paraformaldehyde. The upper side of the membrane was wiped with a cotton ball and the membrane was stained with hematoxylin and removed. The number of migrated cells was counted in six random high-power $(\times 100)$ fields.

\section{ILK cDNA constructs and transfection}

The cDNA constructs wild-type ILK (WT-ILK) and a dominant negative ILK containing the E359K mutation (DN-ILK) and a control plasmid (pUSEamp) were obtained from Upstate (ILK cDNA allelic pack). Transient transfections were performed using Lipofectamine and the Plus-Reagent (Invitrogen, Carlsbad, CA) according to the manufacturer's protocol. Green fluorescent protein (pGFP) was transfected for detection by fluorescence microscopy.

\section{Animals}

ApoE-/- mice on a C57BL/6 background were purchased from Jackson Laboratory (Bar Harbor, ME). C57BL/6J mice (8 weeks old) were purchased from Shanghai SLAC Laboratory Animal Co., Ltd, China.

\section{Atherosclerosis model}

For the atherosclerosis model, mice at 4 weeks of age were shifted from chow to a high fat diet $(0.15 \%$ cholesterol, $21 \%$ fat, and $0.5 \%$ sodium cholate) for 12 weeks.

Cross sections of the aortic root were prepared. After the indicated time period on a high cholesterol diet, mice were sacrificed with ether anesthesia and perfused with $4 \%$ PFA in $0.9 \%$ saline. The entire aorta from the root, extending 5 to $10 \mathrm{~mm}$ after bifurcation of the iliac arteries and including the subclavian, right, and left carotid arteries was dissected and opened longitudinally. Serial cryostat sections of 6- $\mu$ m thickness were prepared.

\section{Injection of tripterine-treated EPCs via the tail vein}

Cells were cultured for 7 days and divided into two groups, namely ox-LDL and tripterine treated groups. Cells were digested by $0.25 \%$ trypsin, centrifuged and resuspended in PBS. The concentration of cells was adjusted to $1 \times 10^{7} / 500 \mu \mathrm{L}$. Atherosclerosis model mice were divided into three groups: group A was the control group (AS, $n=5)$; group B (AS+O-EPCs, $n=5)$ was injected with $1 \times 10^{7}$ ox-LDL treated EPCs through the tail vein immediately after the completion of the model, at $24 \mathrm{~h}$ and $48 \mathrm{~h}$; group $\mathrm{C}$ was the tripterine group (AS+T-EPCs, $n=5$ ), in which mice were injected with $1 \times 10^{7}$ tripterine treated EPCs via the tail vein at the same time points. After 4 weeks, the aorta was dissected and stored at $-80{ }^{\circ} \mathrm{C}$ until assayed.

\section{RNA isolation and qRT-PCR}

For analysis of tripterine-induced changes in ILK mRNA expression, total RNA was extracted from EPCs using the Trizol reagent (Invitrogen) according to the manufacturer's protocol. Reverse transcription was performed using the PrimeScript RT reagent kit (TaKaRa, Dalian China) according to the manufacturer's instructions. For qRT-PCR, cDNA aliquots were amplified using SYBR Premix Ex Taq (TaKaRa). PCR reactions were performed in triplicate. The sequences of the primers used to amplify specific gene products were as follows: ILK sense, 5' - CCA GGT GGC AGA GGT AAGTA-3'; ILK antisense, 5'- CAA GGA ATA AGG TGA GCT TCA GAA-3'.

Immunofluorescence staining of GFP+/CD31+ double positive cells in vessels

The number of EPCs co-expressing GFP and CD31 in vessels of AS and tripterine treated EPCtransplanted AS mice was counted in immunofluorescence stained sections. Arterial sections were generated as described above, blocked and incubated overnight with anti-CD31 antibody (1:200, Santa Cruz Biotechnology, Santa Cruz, CA). After washing in PBS, sections were incubated with fluorescent conjugated secondary antibodies and double labeled immunofluorescence stained sections were evaluated by fluorescence microscopy.

\section{KARGER}


Construction and packaging of rAAV-GFP vectors, cell transduction and mouse model

The rAAV-GFP vector was constructed and packaged as described previously [19]. Briefly, the vector pTR-CBA-Ob EcoRI fragment of pCR-rOb encoding the GFP gene was subcloned into the rAAV vector plasmid pAAV $\beta$ GEnh after deleting the EcoRI fragment. Recombinant AAV particles were generated in HEK293T cells using the AAV Helper-Free system (Stratagene, USA). Cells were transfected with the vector plasmid and cultures were maintained for $54 \mathrm{~h}$ at $37^{\circ} \mathrm{C}$ in DMEM supplemented with $10 \%$ FBS. Cells were collected by centrifugation at $200 \mathrm{~g}$ for $10 \mathrm{~min}$ and pellets were resuspended in $1 \mathrm{ml}$ of PBS, subjected to four freezethaw cycles, and lysates were incubated with $50 \mathrm{U} / \mathrm{ml}$ of Benzonase at $37^{\circ} \mathrm{C}$ for 30 min to digest cellular nucleic acids. After removing cell debris by centrifugation, supernatants (viral stock) were aliquoted and stored at $-70^{\circ} \mathrm{C}$ until use. For cell transduction, EPCs were cultured in dishes, washed with PBS and incubated with 1-2 ml of viral lysate in medium without stem cell growth supplement (CGS) (HyClone, USA). After $3 \mathrm{~h}$ of infection, medium containing 20\% CGS was added to a final concentration of $10 \%$ CGS and infected cells were cultured for $48 \mathrm{~h}$.

EPCs transduced by rAAV-GFP were transplanted into AS rats. Treatment groups (5-6 rats/group) were as follows: 1) untreated EPCs control AS rats 2) tripterine treated EPCs AS rats. A total of $1 \times 10^{7}$ rAAVGFP EPCs and tripterine treated rAAV-GFP EPCs were injected into AS model mice through the tail vein.

Small interfering RNA against ILK

For small interfering RNA (siRNA) targeting ILK, cells were transfected with siRNA duplexes for 48 h using Lipofectamine RNAimax transfection reagent (Invitrogen). The sequence of siRNA targeting ILK was as follows: sense, 5'- GUAGUGUAAUGAUCGAUGAtt-3'; antisense, 5'-UCAUCGAUCAUUACACUACgg-3'. Negative control siRNA was obtained from Applied Biosystems.

\section{Western Blot Analysis}

Western blotting was performed using routine protocols. Briefly, total cell lysates were separated on $10 \%$ sodium dodecyl sulfate polyacrylamide gel electrophoresis and proteins were transferred to polyvinylidene difluoride membranes. After washing and blocking in PBS containing 5\% nonfat dry milk, membranes were incubated in the indicated primary antibodies (all purchased from Abcam): ILK(1:1000), Akt (1:1000), p-Akt (S473) (1:800), GSK3 $3(1: 1000)$, p-GSK3 $\beta(S 9)$ (1:800), catenin (1:1000), cyclin D1 $(1: 1000)$ followed by incubation in horseradish-peroxidase conjugated secondary antibodies. Anti- $\beta$-actin (1:2000, Abcam) was used as the loading control. Bands were detected using enhanced chemiluminescence.

\section{Immunohistochemistry}

For morphological examination, specimens were fixed in $10 \%$ neutral buffered formalin, stained with hematoxylin \& eosin (H\&E), and evaluated by light microscopy. Aortic sections prepared as described above were incubated with anti-ILK antibody followed by biotin-conjugated goat anti-mouse antibody and exposure to avidin-peroxidase complex (Dako, Santa Barbara, CA, USA). Stain development was performed by addition of diaminobenzidine substrate (DAB; Dako) and counterstaining with Mayer's hematoxylin.

\section{Statistical analysis}

Data were expressed as mean \pm SEM for continuous variables and as number (percent) for categorical variables. Comparisons were performed by Student's t test or one-way analysis of variance. Values with p $<0.05$ were considered statistically significant. SPSS 9.0 (version 12, SPSS, Chicago, Illinois, USA) software was used for all statistical analyses.

\section{Results}

Tripterine regulates ILK expression in EPCS

The characterization of bone marrow derived EPCs was described previously (Lu et al. 2014). The effect of tripterine on EPCs was assessed after pre-treatment of cells with oxidized low-density lipoprotein (ox-LDL). Ox-LDL is taken up by EPCs in a receptor-dependent manner and generates oxidative stress through the activation of NADPH oxidase, uncoupling of endothelial nitric oxide synthase (eNOS) and generation of lipid peroxide radicals within 
the plasma membrane, leading to cell necrosis or apoptosis and thus impairing EPC function [20]. The efficiency of ILK knockdown in EPCs was determined by qRT-PCR in cells treated with or without ox-LDL $(200 \mu \mathrm{g} / \mathrm{ml})$ and tripterine $(2.5 \mu \mathrm{M}$ for $4 \mathrm{~h})$. ILK siRNA significantly down regulated ILK in untreated controls and ox-LDL treated EPCs, whereas tripterine partially restored ILK levels (Fig. 1A). The siRNA mediated downregulation of ILK at the protein level was confirmed by western blotting (Fig. 1B). Pretreatment of EPCs with ox-LDL significantly reduced ILK levels, whereas treatment with tripterine restored ILK levels in a dose-dependent manner (Fig. 1C).

Tripterine preconditioning improves ox-LDL treated EPC proliferation, tube formation, migration, and adhesion and reduces cell apoptosis in vitro

The effect of tripterine on EPC neovascularization and migration was determined by in vitro angiogenesis and migration assays in EPCs treated with ox-LDL $(200 \mu \mathrm{g} / \mathrm{ml})$ for $2 \mathrm{~h}$ followed by incubation in tripterine $(2.5 \mu \mathrm{M})$ for $4 \mathrm{~h}$. The results showed that while ox-LDL caused an approximately 2.5 -fold reduction in the number of branch points per high-power field and a 2.25 -fold reduction in the number of migrating cells, tripterine restored the number of vessels and EPC migration to almost control levels (Fig. 2A and B). Knockdown of ILK by siRNA or dominant negative (DN) ILK transfection significantly reduced neovascularization of EPCs and the migratory ability of EPCs in vitro $(\mathrm{p}<0.05)$. Treatment with ox-LDL significantly inhibited cell proliferation as determined by the EdU incorporation assay and Ki67 immunohistochemical staining, whereas tripterine significantly restored proliferation to control levels (Fig. 2C and D). SiRNA or DN-mediated ILK knockdown slightly inhibited EPC proliferation, although the difference did not reach statistical significance in the EdU

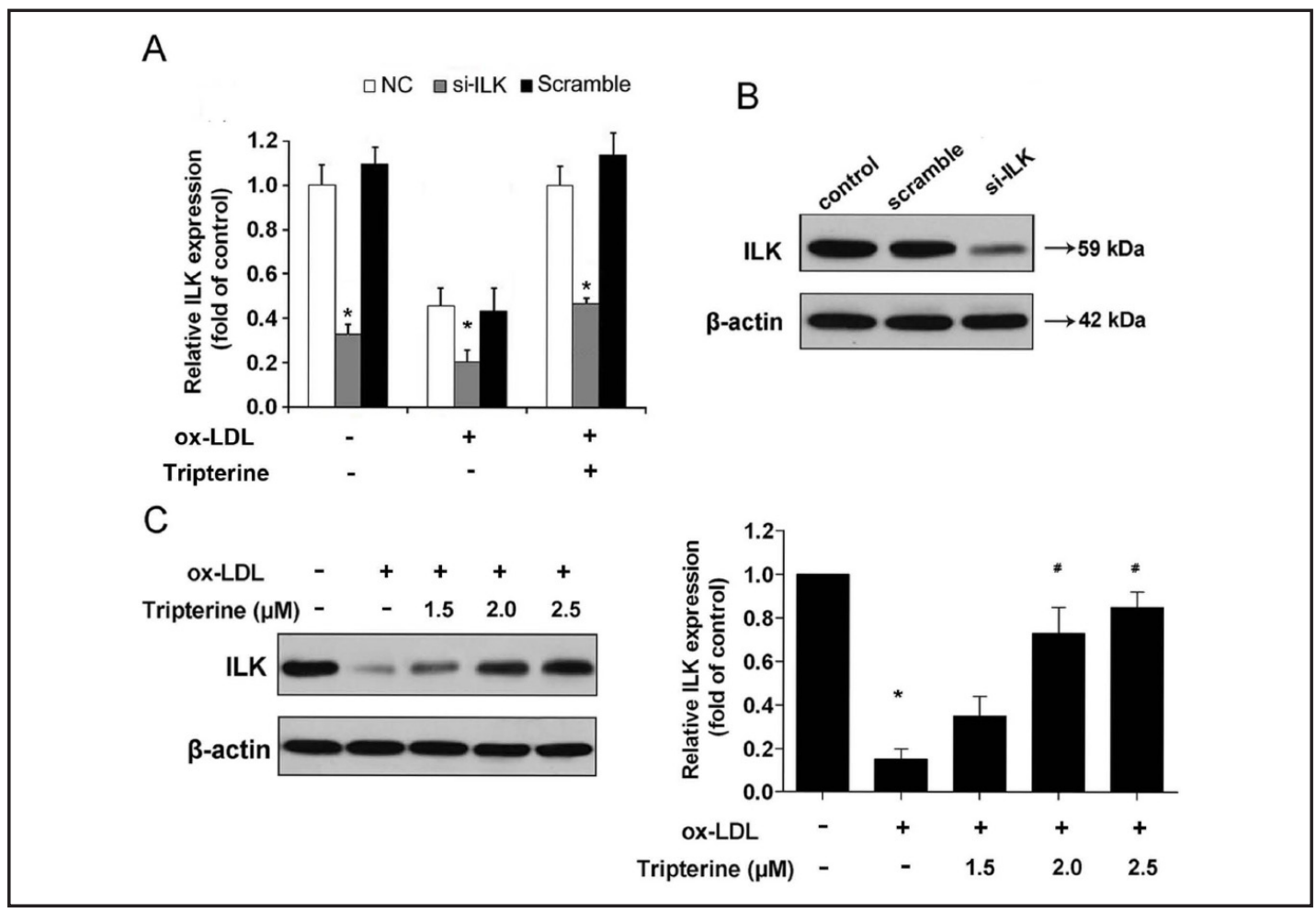

Fig. 1. Tripterine regulates integrin-linked kinase (ILK) expression in EPCs. (A) The efficiency of ILK knockdown was evaluated by qRT-PCR in EPCs treated with or without ox-LDL (200 $\mu \mathrm{g} / \mathrm{ml}$ for $2 \mathrm{~h}$ ) and tripterine $(2.5 \mu \mathrm{M}$ for $4 \mathrm{~h})$. Data are expressed as mean \pm SEM $\left(\mathrm{n}=3,{ }^{*} \mathrm{p}<0.05\right.$, compared with control). (B) Western blot analysis of ILK expression in EPCs treated with shRNA against ILK. (C) Western blot detection of ILK protein expression in EPCs treated with ox-LDL $(200 \mu \mathrm{g} / \mathrm{ml}$ for $2 \mathrm{~h})$ and different concentrations of tripterine (for $4 \mathrm{~h}$ ) ${ }^{*} \mathrm{p}<0.05$, compared with no treatment group; * $\mathrm{p}<0.05$, compared with ox-LDL group). 
Fig. 2. Effects of tripterine treatment and ILK modulation on EPC tube formation, migration, proliferation and adhesion. After incubation with $200 \mu \mathrm{g} / \mathrm{ml}$ ox-LDL for $2 \mathrm{~h}$, cultured EPCs were preconditioned with $2.5 \mu \mathrm{M}$ tripterine for $4 \mathrm{~h}$, and then functional assays were performed. (A) An in vitro angiogenesis assay was used to investigate the effect of tripterine on EPC neovascularization. Representative photomicrographs show the number of branch points in control, ox-LDL treated, tripterine treated, vector, si-ILK or DNILK EPCs (a-f, respectively). (B) A Boyden chamber assay was used to evaluate the effect of tripterine on EPC migration. Migration of control, ox-LDL treated, tripterine treated, vector, si-ILK or DN-ILK EPCs (g-l, respectively) was assessed by hematoxylin staining. (C) Quantification of EPCs 48 $\mathrm{h}$ after tripterine treatment or transfection with control vector, Si-ILK or DN-ILK shown as \% of control. (D) Cell proliferation was assessed by Ki-67 immunocytochemistry in EPCs $48 \mathrm{~h}$ after tripterine treatment or transfection with control vector, si-ILK or DN-ILK. The results are expressed as a percent of the control. (E) A fibronectin adhesion assay was used to evaluate the effect of tripterine and ILK modulation on EPC adhesive function. Data are expressed as mean \pm SEM. $(n=4$, ${ }^{*} \mathrm{p}<0.05$, compared with control group; ${ }^{*} \mathrm{p}<0.05$ compared with ox-LDL group; ’ $\mathrm{p}<0.05$, compared with vector group; ${ }^{\wedge} \mathrm{p}<0.01$, compared with vector group).

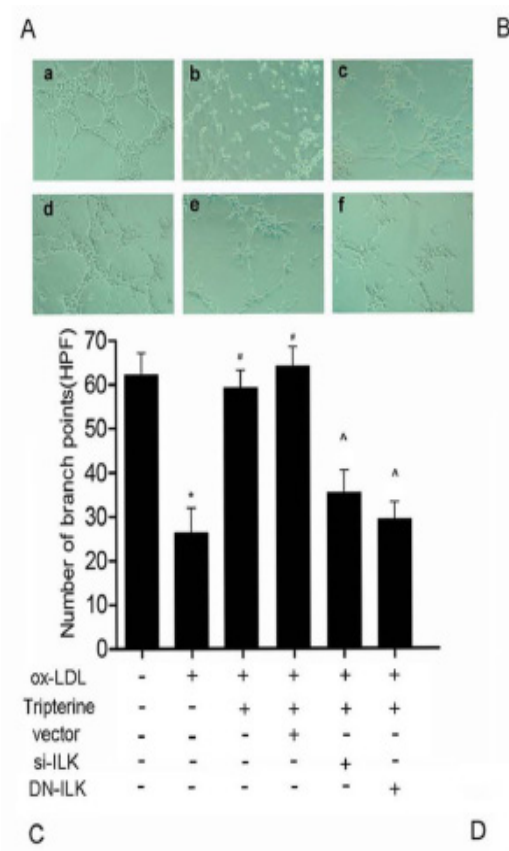

B
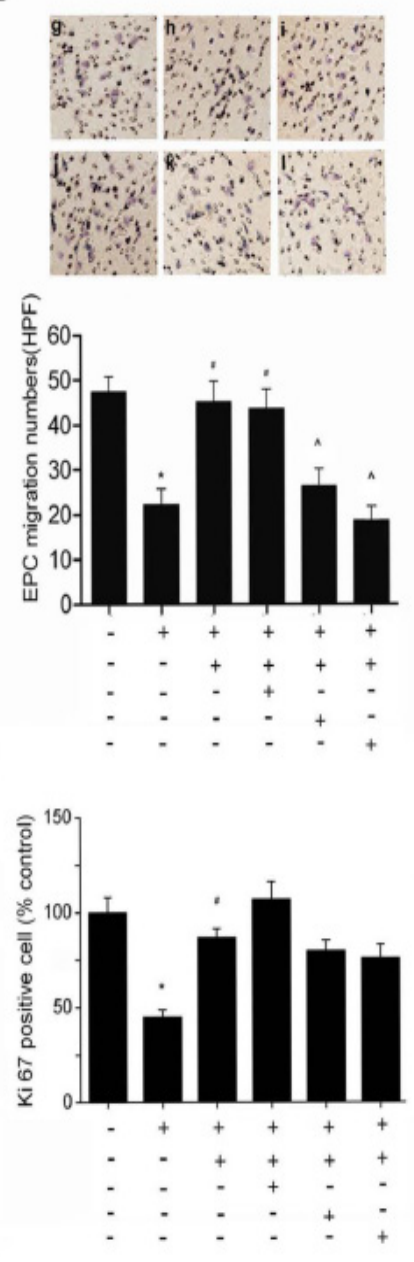

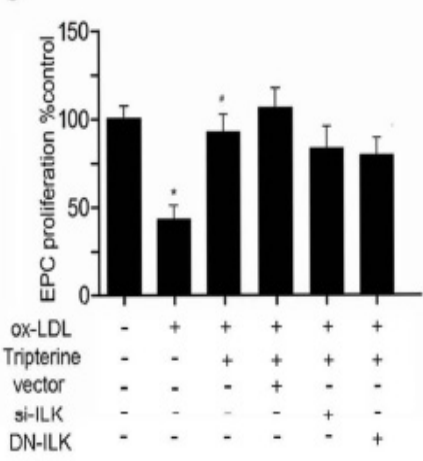

$\mathrm{E}$

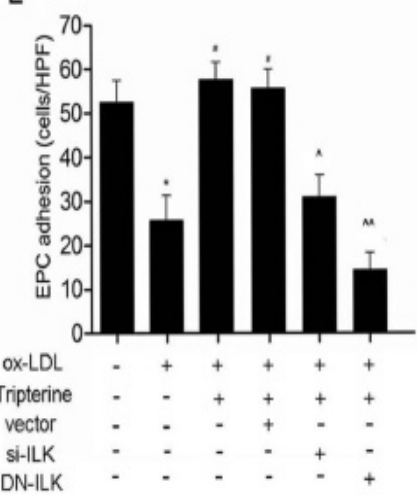

incorporation assay or according to the number of Ki67 positive cells (Fig. 2C and D). siILK and DN-ILK significantly impaired EPC adhesion as determined by fibronectin adhesion assay ( $\mathrm{p}<0.05$ for si-ILK and $\mathrm{p}<0.01$ for DN-ILK) (Fig. 2E). Tripterine treatment significantly restored the ox-LDL induced reduction of the adhesive ability of EPCs $(\mathrm{p}<0.05)$. Apoptosis assessment by TUNEL assay and cleaved caspase 3 detection showed that tripterine reversed the ox-LDL induced increase in the rate of apoptosis in EPCs, whereas si-ILK and DN-ILK had no significant effect on the rate of apoptosis (Fig. 3A and B). 
A
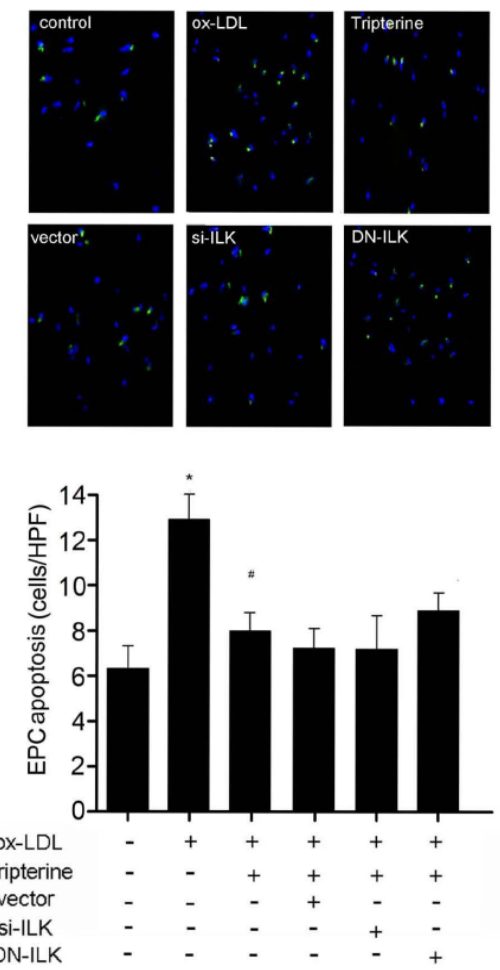

B
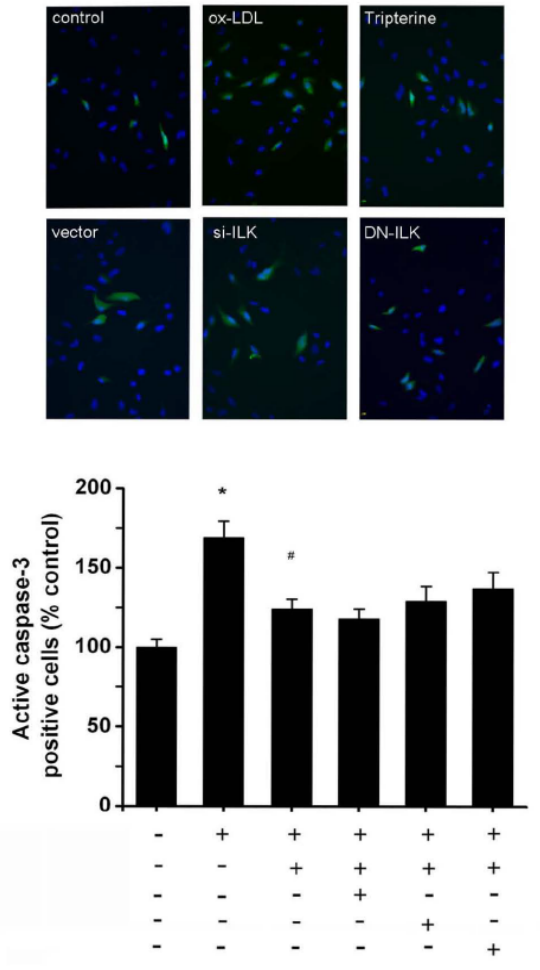

Fig. 3. Effects of tripterine treatment and ILK modulation on EPC apoptosis. (A) The TUNEL assay was used to determine the apoptotic effect of tripterine treatment. Data are expressed as mean \pm SEM. (B) Apoptosis was assessed by immunocytochemical detection of cleaved (active) caspase 3 . $\left(n=4,{ }^{*} \mathrm{p}<0.05\right.$, compared with control group; ${ }^{\#} \mathrm{p}<0.05$ compared with ox-LDL group) Tripterine treatment restored ox-LDL-induced apoptosis, whereas silencing of ILK had no significant effect on EPC apoptosis.

Fig. 4. Immunofluorescence staining of GFP+/CD31+ double positive cells in vessels of AS mice and EPC transplanted AS mice. Tripterine increased the number of GFP+ cells co-localizing with CD31+ cells in vessels ( $\mathrm{n}=5$ mice per group, ${ }^{*} \mathrm{p}<0.05$. compared with AS control).

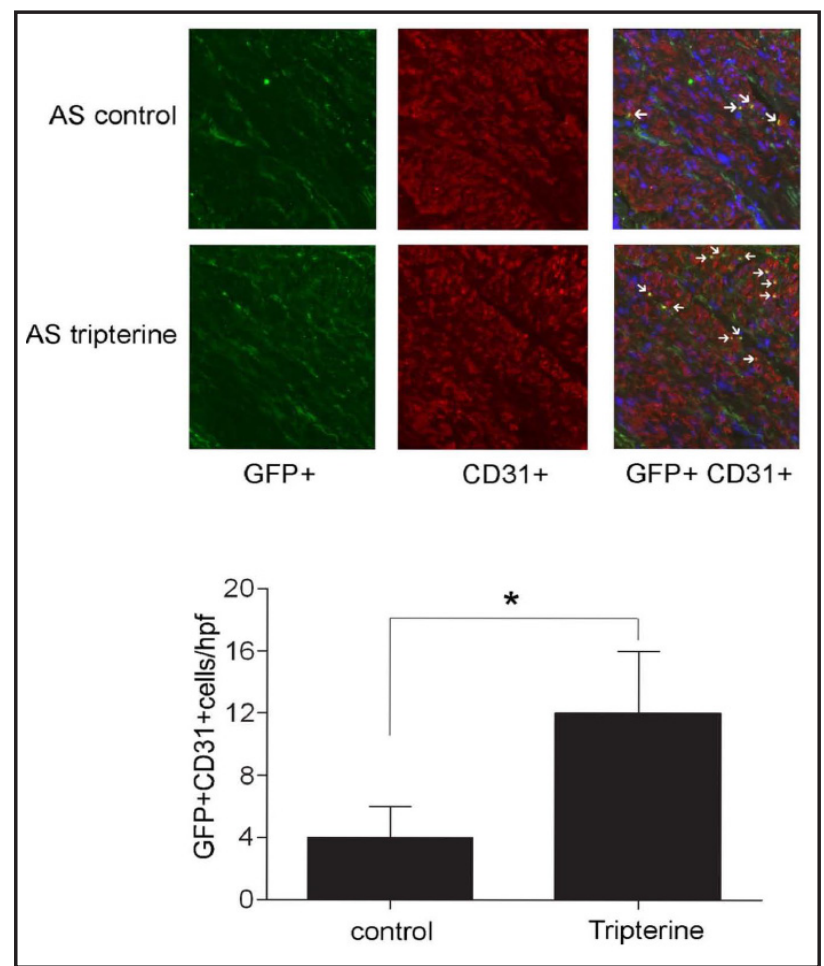




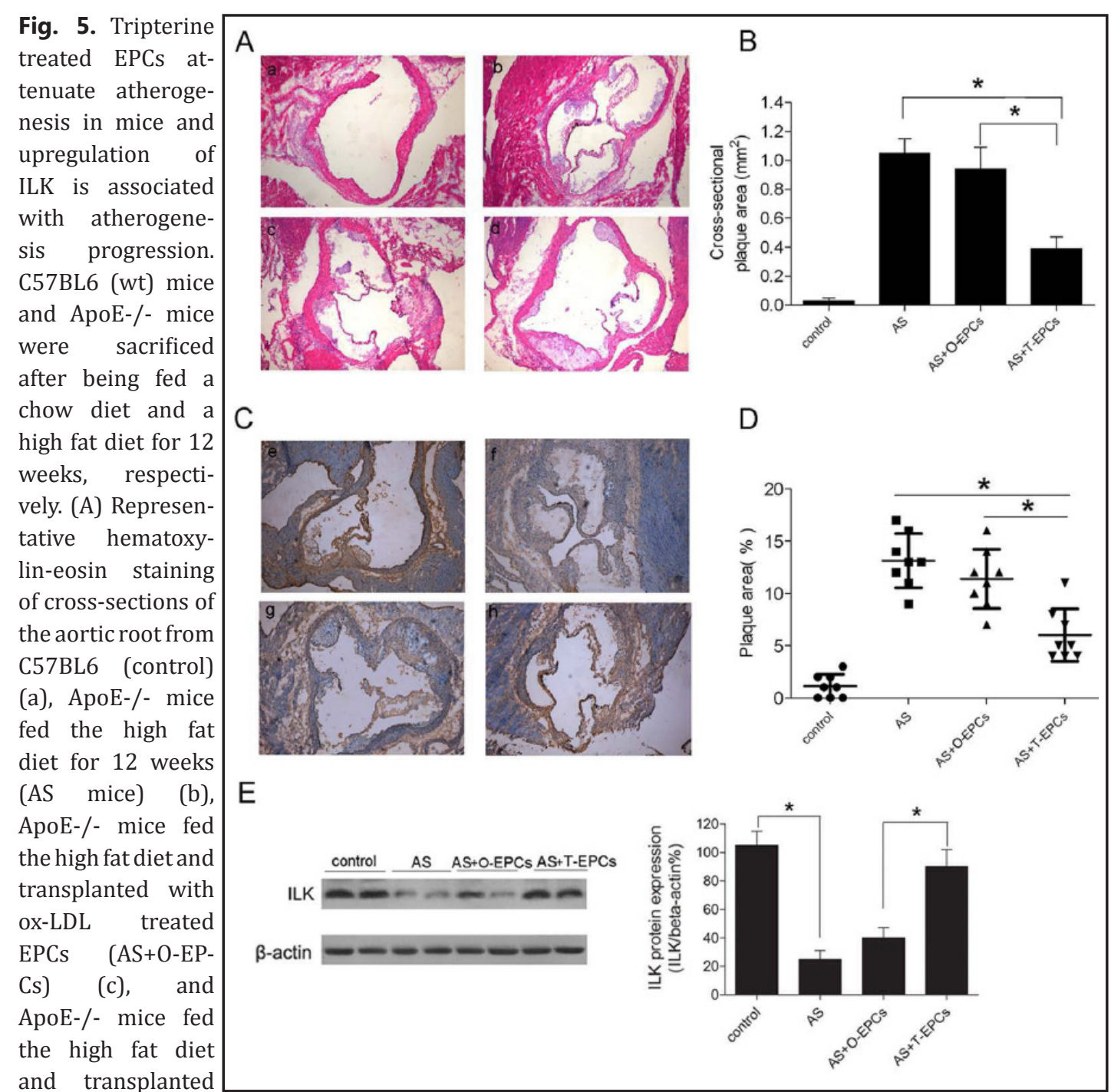

with tripterine treated EPCs (AS+T-EPCs) (d) (100×). (B and C) Quantification of plaque areas in the aortic root (B) and ratios of the lesion area over the aortic media (C) of C57BL6 (control), AS mice, AS+O-EPCs mice and AS+T-EPCs mice. Individual values and means of each group are shown. (D) Representative immunohistochemical staining for ILK (brown) in the aortic root from C57BL6 mice (control) (e), AS mice (f), AS+O-EPCs mice (g), and AS+T-EPCs mice (h) (100x). (E) Western blot detection of ILK expression in the aortic tissues extracted from C57BL6 mice, atherosclerotic mice (AS) and atherosclerotic mice transplanted with tripterine treated EPCs (AS+T-EPCs), with $\beta$-actin as loading control, $n=4$, mean \pm SEM, ${ }^{*} p<0.05$ compared with the AS group.

Tripterine increases the number of GFP+ cells co-localizing with CD31 in vessels

To examine the effect of tripterine in a mouse model of atherosclerosis, mice fed a high cholesterol diet (AS mice) were injected with green fluorescent protein (GFP)-labeled EPCs treated or not with tripterine and the colocalization of GFP with the vascular endothelial marker CD31 was visualized in aortic cross sections by immunofluorescence. The results showed that tripterine increased the number of GFP+ cells colocalizing with CD31+ cells in the vessels of AS mice $(\mathrm{p}<0.05)$, indicating improved incorporation of EPCs into vessels (Fig. 4). 


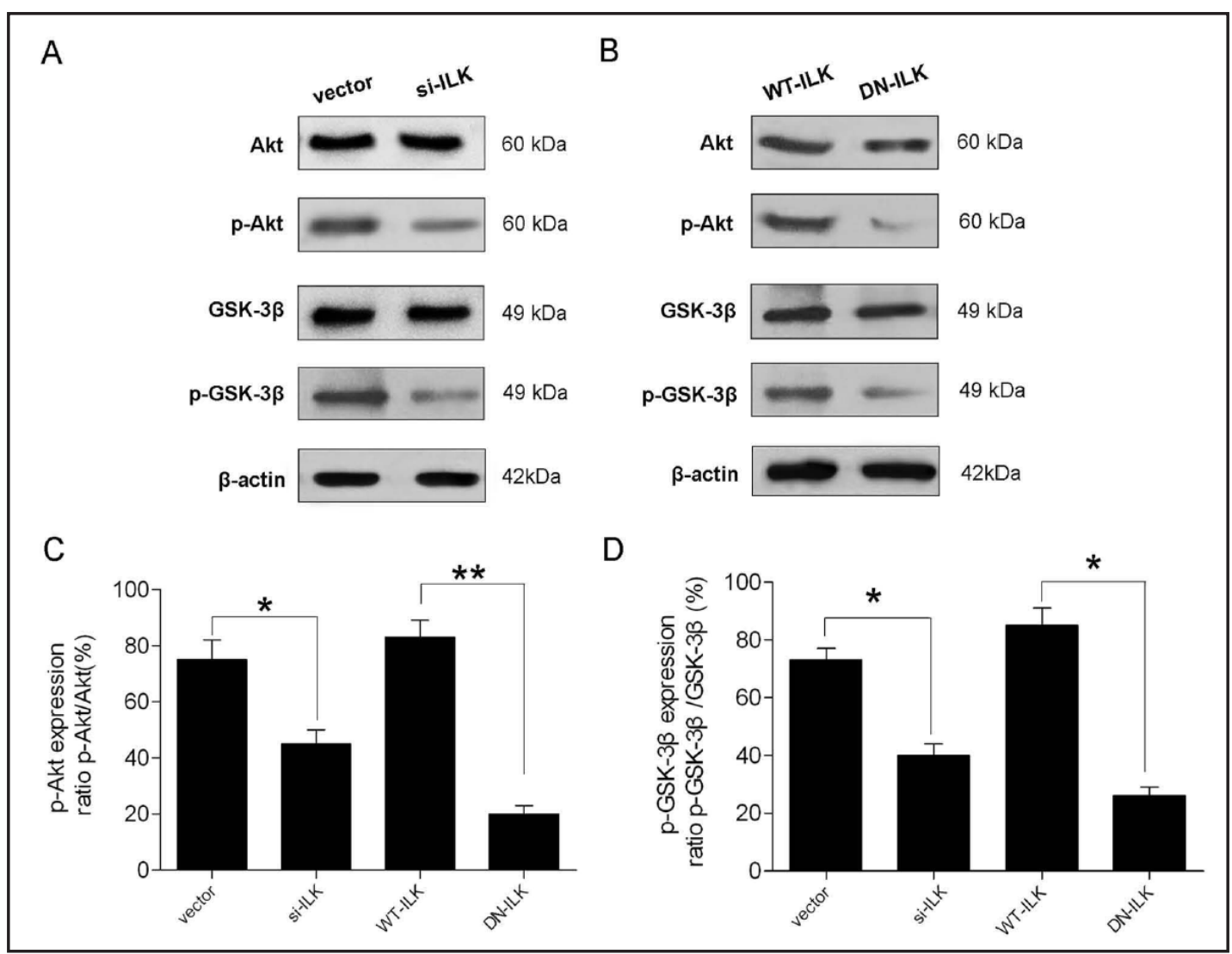

Fig. 6. ILK regulates the Akt/GSK-3 $\beta$ pathway in vitro. (A and B) Western blot assessment of Akt phosphorylation and GSK-3 $\beta$ phosphorylation in si-ILK transfected EPCs and dominant-negative ILK (DN-ILK) EPCs compared to vector and WT-ILK controls. $\mathrm{C}$ and D show the results of densitometric quantification of the western blots in A and B, respectively. ${ }^{*} \mathrm{p}<0.05,{ }^{* *} \mathrm{p}<0.01$.

\section{Tripterine treated EPCs attenuate atherogenesis in mice}

To determine the effect of tripterine on atherogenesis in AS mice, cross sections of the aortic root of mice fed a high fat diet and injected or not with ox-LDL treated EPCs or tripterine treated EPCs were examined by hematoxylin-eosin staining. The results showed a dramatic increase in aortic lesions and plaque formation in the aorta of AS mice compared to that of control mice, which was significantly reduced by pre-treatment of EPCs with tripterine. Figure 5A shows representative H\&E stained cross-sections of the aortic root, and Fig. 5B shows the quantification of the plaque areas. The ratio of the lesion area to the media of the aorta is shown in Fig. 5C, which confirms the significant reduction of plaque area in mice injected with tripterine-treated EPCs. Assessment of ILK expression in the aortic root of AS mice treated as indicated above by immunohistochemistry and western blotting showed a significant downregulation of ILK in AS mice (Fig. 5D and E). However, AS mice transplanted with tripterine treated EPCs showed partial restoration of ILK levels, as seen in representative sections of immunohistochemical staining for ILK (Fig. 5D) and western blotting results (Fig. 5E). Figure 5D (f) and (g) show cross sections of the aorta from AS and AS+O-EPC mice, illustrating the downregulation of ILK compared to the control in panel e, which is restored in tripterine-EPC mice in panel h. Fig. 5E shows representative western blots and quantification by densitometry.

\section{ILK regulates Akt/GSK-3 $\beta$ phosphorylation and the expression of $\beta$-catenin and cyclin D1} in vitro

We next examined the effect of ILK silencing on the activation of its downstream signaling targets in EPCs. The results of western blot analysis showed that knockdown of 


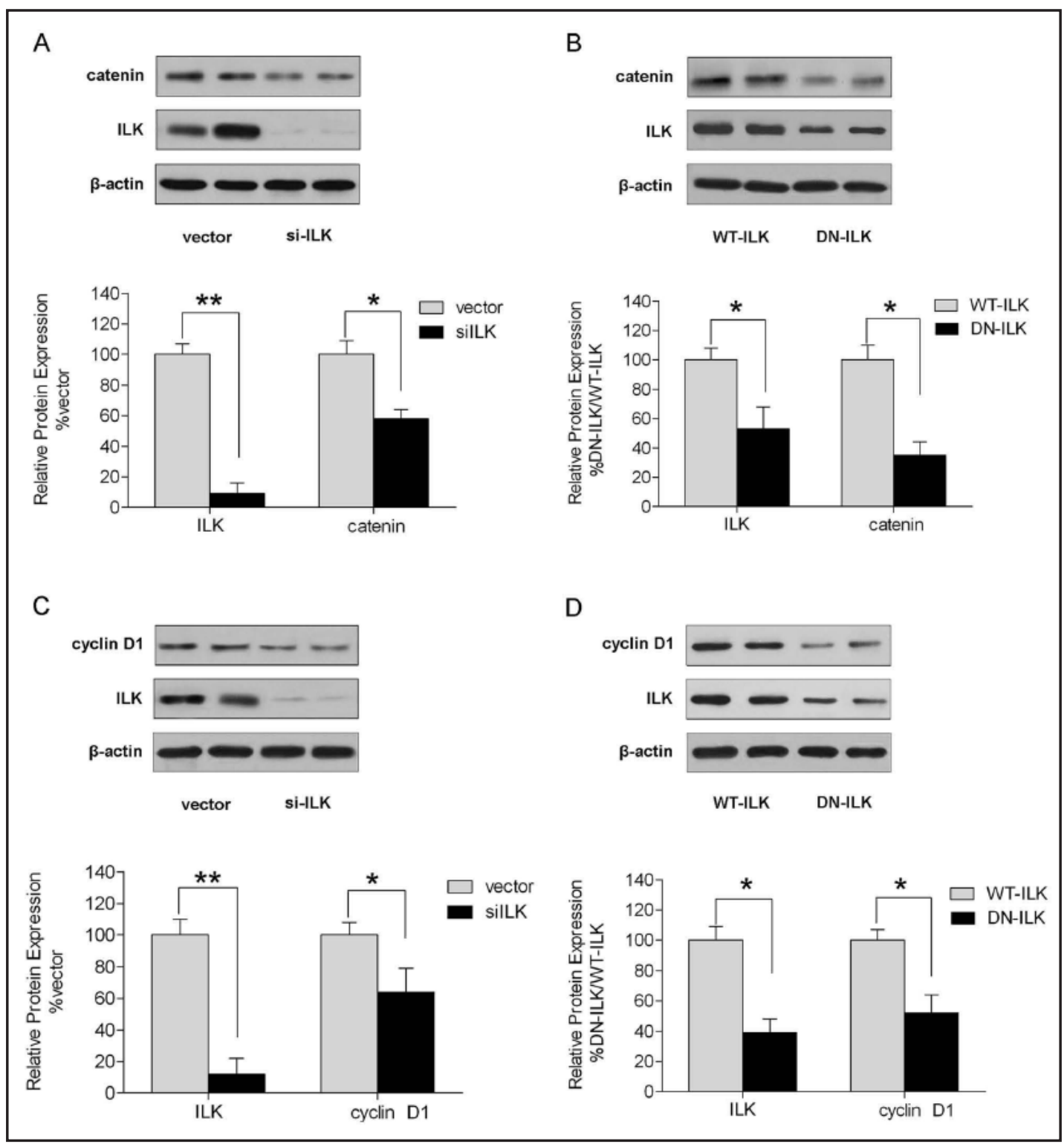

Fig. 7. ILK modulation of $\beta$-catenin and cyclin D1 expression. Western blot assessment of the effect of si-ILK and DN-ILK on the expression of $\beta$-catenin (A and B) and cyclin D1 (C and D) and quantification by densitometry. Results are expressed as relative to the vector or WT-ILK controls. ${ }^{*} \mathrm{p}<0.05,{ }^{* *} \mathrm{p}<0.01, \mathrm{n}=5$.

ILK by siRNA or DN-ILK significantly decreased the levels of phospho Akt (p-Akt) and p-GSK$3 \beta$ (Fig. 6A-D). Knockdown of ILK by si-ILK or DN-ILK also significantly down regulated $\beta$-catenin and cyclin D1 compared to vector or WT ILK (Fig. 7A-D).

\section{Discussion}

In atherosclerosis, the deposition of LDL in the vascular endothelium and the influx of inflammatory leukocytes into the vascular wall result in the formation of atherosclerotic plaques associated with several cardiovascular diseases [2]. The integrity of the endothelial monolayer, which plays an important role in atherogenesis, can be compromised by endothelial cell damage, and the balance between the magnitude of injury and the capacity for repair predicts the risk of cardiovascular events [21]. The development of therapies that KARGER 
promote endothelial repair and atherosclerosis regression, a process that remains poorly understood, is of interest. Bone marrow derived EPCs play an important role in endothelial cell regeneration and vascular repair [22], and reduced levels of circulating EPCs are predictive of atherosclerotic disease progression and cardiovascular events [23, 24]. EPC transplantation has been investigated for the treatment of cardiovascular diseases. However, the success of EPC transplantation depends on the functional status of the transplanted progenitor cells, and the mechanisms of EPC dysfunction remain unclear. In the present study, we showed that tripterine improves EPC function via a mechanism involving ILK and its downstream effectors Akt, GSK-3 $\beta$, $\beta$-catenin and cyclin D1 in vitro, and investigated its effectiveness in vivo in a mouse model of atherosclerosis. Our findings suggest a potential therapeutic value of this triterpenoid compound for the treatment of atherosclerosis.

The capacity of EPCs to differentiate into endothelial cells and participate in vascular repair and an extensive body of research showing that dysfunctional EPCs have defects in angiogenic properties and can contribute to vascular and cardiovascular diseases underscores the importance of developing strategies to improve EPC function $[25,26]$. Genetic modification of EPCs with vascular endothelial growth factor (VEGF) improved their proliferation, adhesion and incorporation into endothelial cell monolayers, and improved neovascularization and blood flow recovery in a mouse model [27]. Granulocyte colonystimulating factor increases the mobilization of EPCs into the peripheral blood, which has been proposed as strategy for the prevention of ischemic heart disease and myocardial infarction $[28,29]$. The mobilization of EPCs has been suggested as a strategy for the regression of atherosclerosis. PPAR- $\gamma$ agonists increase the mobilization of bone-marrow-derived progenitor cells through a mechanism involving the Akt pathway [30]. EPC mobilization by treatment with AMD3100 and transfer of bone marrow EPCs was shown to improve plaque regression in a mouse model of atherosclerosis [2]. Several strategies aimed at improving autologous EPC function by ex vivo conditioning with growth factors/chemoattractants such as SDF-1 $\alpha$, VEGF, and interleukin-8, antioxidants, hormones and clinically available drugs such as statins and ACE-inhibitors have been shown to improve EPC function [26]. In the present study, we explored the effect of tripterine on ox-LDL induced EPC dysfunction and showed its ability to increase proliferation, migration and the adhesive abilities of EPCs as well as attenuating ox-LDL induced EPC apoptosis._Furthermore, tripterine improved the incorporation of EPCs into vessels and decreased atherogenesis in atherosclerotic mice, suggesting its potential for the improvement of EPC function.

In the present study, tripterine treatment reversed the ox-LDL induced downregulation of ILK in EPCs, and transplantation of tripterine-treated EPCs restored ILK levels in AS mice. The role of ILK as a regulator of blood vessel integrity and its involvement in angiogenesis, matrix-endothelial cell interaction and the recruitment of endothelial progenitors has been shown previously [10,31]. ILK is down regulated in the endothelium of atherosclerotic blood vessels, suggesting that it plays a role in pathological vascular remodeling in atherosclerosis [32]. Disruption of the interaction of ILK with eNOS and HSP90 was suggested to underlie endothelial dysfunction in atherosclerosis. Furthermore, the stabilization of ILK by HSP90 was shown to play a role in the homing of EPCs to ischemic tissues [33]. We previously showed that tripterine enhances EPC function through the induction of HSP32 [18], which taken together with the present findings and prior reports linking HSP90 to ILK, indicates that heat shock proteins may play a role in the stabilization of ILK in relation to EPC dysfunction and further investigation is warranted.

Our findings showing the regulatory role of ILK in EPCs are supported by previous studies. SiRNA mediated silencing of ILK inhibits endothelial cell migration, proliferation and tube formation [34], and restoration of ILK expression in EPCs from patients with coronary artery disease rescues the migratory ability of EPCs via the modulation of cyclin D1 signaling and the actin cytoskeleton [35]. We showed that knockdown of ILK impaired EPC vascularization, migration and adhesion and confirmed that ILK acts by modulating cyclin D1. However, ILK silencing had no effect on EPC proliferation, which differs from the results of Werner et al. Nevertheless, these studies suggest ILK as a therapeutic target in endothelial

\section{KARGER}


dysfunction related diseases such as atherosclerosis and thus highlight the importance of our present findings showing the modulation of ILK by tripterine.

The effects of tripterine on EPC function in the present study were mediated by the ILK/ Akt signaling pathway. The Akt pathway has been shown to mediate the effects of statin on EPCs by enhancing their proliferative and migratory activities [30]. Akt plays a role as a mediator of angiogenesis by regulating the migration, survival, proliferation and tubulogenesis of vascular endothelial cells [36]. In the present study, siRNA mediated silencing of ILK down regulated the expression of $\beta$-catenin and cyclin D1. ILK, through the phosphorylation of its downstream effectors Akt and GSK-3 $\beta$, is an important modulator of several signaling pathways including the canonical Wnt/ $\beta$-catenin pathway, which regulates the expression of cell cycle genes including cyclin D1 $[37,38]$. ILK was previously shown to be involved in intimal thickening associated with atherosclerosis by modulating vascular smooth muscle cell proliferation and migration through the regulation of cell-matrix contacts and $\beta$-catenin signaling [39]. We showed that silencing of ILK inhibited Akt and GSK-3 $\beta$ phosphorylation and down regulated $\beta$-catenin and cyclin D1, and tripterine treatment restored ILK levels, suggesting a possible mechanism by which tripterine improves EPC function.

In the present study, tripterine treatment suppressed ox-LDL induced apoptosis in EPCs. Based on our data showing that the effects of tripterine are mediated by the ILK/ Akt/GSK-3 $\beta$ pathway, its effect on apoptosis could have been mediated by the upregulation of ILK, leading to the phosphorylation of GSK-3 $\beta$ and the inhibition of the proapoptotic protein Bim $[11,40]$. Another possible mechanism involves the phosphorylation of Akt by ILK leading to the activation of NF- $\kappa B$ and the upregulation of antiapoptotic proteins such as Bcl-xL $[41,42]$. Further experiments examining the involvement of these signaling pathways may improve our understanding of the mechanism of action of tripterine in EPCs.

In summary, we showed that tripterine improved cell proliferation, tube forming ability, migration, and adhesion and inhibited apoptosis of EPCs in vitro. In a mouse model of atherosclerosis, tripterine improved the mobilization of EPCs into vessels, reduced the formation of plaque and improved aortic lesions. The effects of tripterine were shown to be mediated by the modulation of ILK and its downstream effectors Akt, GSK-3 $\beta$, $\beta$-catenin and cyclin D1. Tripterine improved vascular function by enhancing the properties of EPCs, suggesting its potential therapeutic value for the treatment of atherosclerosis.

\section{Acknowledgments}

This work was supported by International Science \& Technology Cooperation Program of China, Grant No.2013DFG32170; Natural Science Foundation of China, Grant No. 81071242; the Program of Science and Technology Commission of Shanghai Municipality 13ZR1414500. Funding agencies had no role in study design, data collection, analysis, decision to publish, or preparation of the manuscript.

\section{Disclosure Statement}

The authors declare no conflict of interest.

\section{References}

1 Hansson GK, Libby P: The immune response in atherosclerosis: A double-edged sword. Nat Rev Immunol 2006;6:508-519.

2 Yao L, Heuser-Baker J, Herlea-Pana O, Iida R, Wang Q, Zou MH, Barlic-Dicen J: Bone marrow endothelial progenitors augment atherosclerotic plaque regression in a mouse model of plasma lipid lowering. Stem Cells 2012;30:2720-2731. 
3 Carmeliet P, Jain RK: Molecular mechanisms and clinical applications of angiogenesis. Nature 2011;473:298-307.

4 Urbich C, Aicher A, Heeschen C, Dernbach E, Hofmann WK, Zeiher AM, Dimmeler S: Soluble factors released by endothelial progenitor cells promote migration of endothelial cells and cardiac resident progenitor cells. J Mol Cell Cardiol 2005;39:733-742.

5 Shantsila E, Watson T, Tse HF, Lip GY: New insights on endothelial progenitor cell subpopulations and their angiogenic properties. J Am Coll Cardiol 2008;51:669-671.

6 Kalka C, Masuda H, Takahashi T, Kalka-Moll WM, Silver M, Kearney M, Li T, Isner JM, Asahara T: Transplantation of ex vivo expanded endothelial progenitor cells for therapeutic neovascularization. Proc Natl Acad Sci U S A 2000;97:3422-3427.

7 Chen X, Gu M, Zhao X, Zheng X, Qin Y, You X: Deterioration of cardiac function after acute myocardial infarction is prevented by transplantation of modified endothelial progenitor cells overexpressing endothelial no synthases. Cell Physiol Biochem 2013;31:355-365.

8 Kawamoto A, Gwon HC, Iwaguro H, Yamaguchi JI, Uchida S, Masuda H, Silver M, Ma H, Kearney M, Isner JM, Asahara T: Therapeutic potential of ex vivo expanded endothelial progenitor cells for myocardial ischemia. Circulation 2001;103:634-637.

9 Hannigan GE, Leung-Hagesteijn C, Fitz-Gibbon L, Coppolino MG, Radeva G, Filmus J, Bell JC, Dedhar S: Regulation of cell adhesion and anchorage-dependent growth by a new beta 1-integrin-linked protein kinase. Nature 1996;379:91-96.

10 Legate KR, Montanez E, Kudlacek O, Fassler R: Ilk, pinch and parvin: The tipp of integrin signalling. Nat Rev Mol Cell Biol 2006;7:20-31.

11 del Nogal M, Luengo A, Olmos G, Lasa M, Rodriguez-Puyol D, Rodriguez-Puyol M, Calleros L: Balance between apoptosis or survival induced by changes in extracellular-matrix composition in human mesangial cells: A key role for ilk-nfkappab pathway. Apoptosis 2012;17:1261-1274.

12 Boulter E, Grall D, Cagnol S, Van Obberghen-Schilling E: Regulation of cell-matrix adhesion dynamics and rac-1 by integrin linked kinase. FASEB J 2006;20:1489-1491.

13 Friedrich EB, Liu E, Sinha S, Cook S, Milstone DS, MacRae CA, Mariotti M, Kuhlencordt PJ, Force T, Rosenzweig A, St-Arnaud R, Dedhar S, Gerszten RE: Integrin-linked kinase regulates endothelial cell survival and vascular development. Mol Cell Biol 2004;24:8134-8144.

14 Friedrich EB, Clever YP, Wassmann S, Werner N, Bohm M, Nickenig G: Role of integrin-linked kinase in vascular smooth muscle cells: Regulation by statins and angiotensin ii. Biochem Biophys Res Commun 2006;349:883-889.

15 Wu F, Han M, Wilson JX: Tripterine prevents endothelial barrier dysfunction by inhibiting endogenous peroxynitrite formation. Br J Pharmacol 2009;157:1014-1023.

16 Gu L, Bai W, Li S, Zhang Y, Han Y, Gu Y, Meng G, Xie L, Wang J, Xiao Y, Shan L, Zhou S, Wei L, Ferro A, Ji Y: Celastrol prevents atherosclerosis via inhibiting lox-1 and oxidative stress. PLoS One 2013;8:e65477.

17 Kang SW, Kim MS, Kim HS, Kim Y, Shin D, Park JH, Kang YH: Celastrol attenuates adipokine resistinassociated matrix interaction and migration of vascular smooth muscle cells. J Cell Biochem 2013;114:398408.

18 Lu C, Zhang X, Zhang D, Pei E, Xu J, Tang T, Ye M, Uzan G, Zhi K, Li M: Short time tripterine treatment enhances endothelial progenitor cell function via heat shock protein 32. J Cell Physiol 2015;230:11391147.

19 Iwaniec UT, Boghossian S, Trevisiol CH, Wronski TJ, Turner RT, Kalra SP: Hypothalamic leptin gene therapy prevents weight gain without long-term detrimental effects on bone in growing and skeletally mature female rats. J Bone Miner Res 2011;26:1506-1516.

20 Tie G, Yan J, Yang Y, Park BD, Messina JA, Raffai RL, Nowicki PT, Messina LM: Oxidized low-density lipoprotein induces apoptosis in endothelial progenitor cells by inactivating the phosphoinositide 3-kinase/akt pathway. J Vasc Res 2010;47:519-530.

21 Huang PH, Chen JS, Tsai HY, Chen YH, Lin FY, Leu HB, Wu TC, Lin SJ, Chen JW: Globular adiponectin improves high glucose-suppressed endothelial progenitor cell function through endothelial nitric oxide synthase dependent mechanisms. J Mol Cell Cardiol 2011;51:109-119.

22 Huang S, Tang Y, Cai X, Peng X, Liu X, Zhang L, Xiang Y, Wang D, Wang X, Pan T: Celastrol inhibits vasculogenesis by suppressing the vegf-induced functional activity of bone marrow-derived endothelial progenitor cells. Biochem Biophys Res Commun 2012;423:467-472. 
23 Dimmeler S, Zeiher AM: Vascular repair by circulating endothelial progenitor cells: The missing link in atherosclerosis? J Mol Med (Berl) 2004;82:671-677.

24 Liu Y, Hao F, Zhang H, Cao D, Lu X, Li X: Panax notoginseng saponins promote endothelial progenitor cell mobilization and attenuate atherosclerotic lesions in apolipoprotein e knockout mice. Cell Physiol Biochem 2013;32:814-826.

25 Loomans CJ, de Koning EJ, Staal FJ, Rookmaaker MB, Verseyden C, de Boer HC, Verhaar MC, Braam B, Rabelink TJ, van Zonneveld AJ: Endothelial progenitor cell dysfunction: A novel concept in the pathogenesis of vascular complications of type 1 diabetes. Diabetes 2004;53:195-199.

26 Petrelli A, Di Fenza R, Carvello M, Gatti F, Secchi A, Fiorina P: Strategies to reverse endothelial progenitor cell dysfunction in diabetes. Exp Diabetes Res;2012:471823.

27 Iwaguro H, Yamaguchi J, Kalka C, Murasawa S, Masuda H, Hayashi S, Silver M, Li T, Isner JM, Asahara T: Endothelial progenitor cell vascular endothelial growth factor gene transfer for vascular regeneration. Circulation 2002;105:732-738.

28 Takahashi T, Kalka C, Masuda H, Chen D, Silver M, Kearney M, Magner M, Isner JM, Asahara T: Ischemia- and cytokine-induced mobilization of bone marrow-derived endothelial progenitor cells for neovascularization. Nat Med 1999;5:434-438.

29 Korbling M, Reuben JM, Gao H, Lee BN, Harris DM, Cogdell D, Giralt SA, Khouri IF, Saliba RM, Champlin RE, Zhang W, Estrov Z: Recombinant human granulocyte-colony-stimulating factor-mobilized and apheresiscollected endothelial progenitor cells: A novel blood cell component for therapeutic vasculogenesis. Transfusion 2006;46:1795-1802.

30 Llevadot J, Murasawa S, Kureishi Y, Uchida S, Masuda H, Kawamoto A, Walsh K, Isner JM, Asahara T: Hmg-coa reductase inhibitor mobilizes bone marrow--derived endothelial progenitor cells. J Clin Invest 2001;108:399-405.

31 Lal H, Verma SK, Foster DM, Golden HB, Reneau JC, Watson LE, Singh H, Dostal DE: Integrins and proximal signaling mechanisms in cardiovascular disease. Front Biosci (Landmark Ed) 2009;14:2307-2334.

32 Herranz B, Marquez S, Guijarro B, Aracil E, Aicart-Ramos C, Rodriguez-Crespo I, Serrano I, Rodriguez-Puyol M, Zaragoza C, Saura M: Integrin-linked kinase regulates vasomotor function by preventing endothelial nitric oxide synthase uncoupling: Role in atherosclerosis. Circ Res 2012;110:439-449.

33 Lee SP, Youn SW, Cho HJ, Li L, Kim TY, Yook HS, Chung JW, Hur J, Yoon CH, Park KW, Oh BH, Park YB, Kim HS: Integrin-linked kinase, a hypoxia-responsive molecule, controls postnatal vasculogenesis by recruitment of endothelial progenitor cells to ischemic tissue. Circulation 2006;114:150-159.

34 Guo L, Yu W, Li X, Zhao G, He P: Targeting of integrin-linked kinase with a small interfering rna inhibits endothelial cell migration, proliferation and tube formation in vitro. Ophthalmic Res 2009;42:213-220.

35 Werner C, Bohm M, Friedrich EB: Role of integrin-linked kinase for functional capacity of endothelial progenitor cells in patients with stable coronary artery disease. Biochem Biophys Res Commun 2008;377:331-336.

36 Dimmeler S, Fleming I, Fisslthaler B, Hermann C, Busse R, Zeiher AM: Activation of nitric oxide synthase in endothelial cells by akt-dependent phosphorylation. Nature 1999;399:601-605.

37 Oloumi A, Syam S, Dedhar S: Modulation of wnt3a-mediated nuclear beta-catenin accumulation and activation by integrin-linked kinase in mammalian cells. Oncogene 2006;25:7747-7757.

38 Persad S, Attwell S, Gray V, Mawji N, Deng JT, Leung D, Yan J, Sanghera J, Walsh MP, Dedhar S: Regulation of protein kinase b/akt-serine 473 phosphorylation by integrin-linked kinase: Critical roles for kinase activity and amino acids arginine 211 and serine 343. J Biol Chem 2001;276:27462-27469.

39 Dwivedi A, Sala-Newby GB, George SJ: Regulation of cell-matrix contacts and beta-catenin signaling in vsmc by integrin-linked kinase: Implications for intimal thickening. Basic Res Cardiol 2008;103:244-256.

40 von Wnuck Lipinski K, Keul P, Ferri N, Lucke S, Heusch G, Fischer JW, Levkau B: Integrin-mediated transcriptional activation of inhibitor of apoptosis proteins protects smooth muscle cells against apoptosis induced by degraded collagen. Circ Res 2006;98:1490-1497.

41 Tan C, Mui A, Dedhar S: Integrin-linked kinase regulates inducible nitric oxide synthase and cyclooxygenase-2 expression in an nf-kappa b-dependent manner. J Biol Chem 2002;277:3109-3116.

42 Hayden MS, Ghosh S: Shared principles in nf-kappab signaling. Cell 2008;132:344-362. 